\title{
Sleep Apnea Identification using HRV Features of ECG Signals
}

\author{
Billy Sulistyo, Nico Surantha, Sani M. Isa \\ Computer Science Department, Binus Graduate Program - Master of Computer Science, \\ Bina Nusantara University, Jakarta, Indonesia 11480
}

\begin{tabular}{l}
\hline \hline Article Info \\
\hline Article history: \\
Received Apr 10, 2018 \\
Revised Jun 21, 2018 \\
Accepted Jul 10, 2018
\end{tabular}

\section{Keyword:}

ECG

Feature extraction

HRV

Sleep apnea

\begin{abstract}
Sleep apnea is a common sleep disorder that interferes with the breathing of a person. During sleep, people can stop breathing for a moment that causes the body lack of oxygen that lasts for several seconds to minutes even until the range of hours. If it happens for a long period, it can result in more serious diseases, e.g. high blood pressure, heart failure, stroke, diabetes, etc. Sleep apnea can be prevented by identifying the indication of sleep apnea itself from ECG, EEG, or other signals to perform early prevention. The purpose of this study is to build a classification model to identify sleep disorders from the Heart Rate Variability (HRV) features that can be obtained with Electrocardiogram (ECG) signals. In this study, HRV features were processed using several classification methods, i.e. ANN, KNN, N-Bayes and SVM linear Methods. The classification is performed using subjectspecific scheme and subject-independent scheme. The simulation results show that the SVM method achieves higher accuracy other than three other methods in identifying sleep apnea. While, time domain features shows the most dominant performance among the HRV features.
\end{abstract}

Copyright $@ 2018$ Institute of Advanced Engineering and Science. All rights reserved.

\section{Corresponding Author:}

Nico Surantha,

Computer Science Department,

Binus Graduate Program - Master of Computer Science,

Bina Nusantara University,

Jakarta, Indonesia 11480.

Email: nsurantha@binus.edu

\section{INTRODUCTION}

Sleep is very important for the human body to perform optimally. During sleep, the body will form and regenerate cells, support brain function, and recharge the body's energy. For children and adolescents, sleep is required to help the growth process. Sleep can be divided into two phases, i.e. rapid eye movement (REM) and non-Rapid Eye Movement (NREM) where both are always repeated in sleep [1]. When a person does not experience normal REM and non-REM cycles, the body will experience various adverse effects such as fatigue, decreasing ability to concentrate, disrupted body metabolism, and so on [2] .

There are various types of sleeping disorder, e.g. insomnia, narcolepsy, sleep apnea, parasomnia, hypersomnia, restless leg syndrome, etc [3]. Sleep apnea is a disturbance to the breathing process because the wall of the throat is relaxed and narrowed while sleeping [4]. While sleeping, the muscles of the throat become relaxed and weak. When the muscles are too weak and not treated immediately, it may cause constriction or even block the airways that potentially cause health problems, accidents, and premature death. There are 3 types of sleep apnea, i.e. obstructive sleep apnea caused by obstruction of the respiratory tract, central sleep apnea caused by the unstable respiratory control centers that result in the brain failing to signal the breathing muscles [4],[5], and mixed complex which is a combination of obstructive and central apnea Sleep apnea is very common, usually found more in men than in women. This condition can occur in patients of any age, but more common in middle-aged adults. Sleep apnea can be treated by knowing the symptoms or signs of sleep apnea, reducing risk factors and being discussed with the doctor for further action. Common 
symptoms are snoring, others notice some breathing interruptions during sleep, sudden wake with shortness of breath, headache in the morning, insomnia, attention problems, irritability, and hypersomnia. Sleep apnea disorder can cause hypertension, high blood pressure, stroke, obesity, and diabetes. Sleep apnea could not be treated, but can be diminished by doing treatments such as behavioral therapy, positive pressure therapy, installation of oral breathing apparatus, and operation [6], [7].

There are some researches have been performed in sleep apnea identification. Almazaydeh, et al., performs obstructive sleep apnea detection using support vector machine (SVM) method [8]. The feature used in the paper are mean epoch, standard deviation epoch, NN50 (variant 1), NN50 (variant 2), pNN50, etc. They used ECG signal database from physionet.org as their data set. On the other hand, Yilmaz, et.al. have proposed sleep stage and obstructive apneaic epoch classification using single-lead ECG in order to classify sleep stage and sleep apnea automatically using single-lead ECG [9]. In this research, Yilmaz perform data preprocessing and feature extraction before the classification. Another paper from Carolina Varon, et al., performs sleep apnea classification using four easily computable features, three generally known ones and a newly proposed feature [10]. They perform classification using least squares support vector machines (LS-SVM) with RBF kernel. Finally, Sani M. Isa, et all proposed the implementation of the principal component analysis (PCA) in sleep apnea identification in order to improve the the accuracy of classification process [11].

According to the study that have been performed in this area, it is interesting to conduct performance evaluation among well known classification method in the similar simulation condition. In this research we evaluate the performance of $\mathrm{ANN}, \mathrm{KNN}, \mathrm{N}$-bayes, and support vector machine in classifying sleep apnea. Then, mostly in previous study, they perform classication for 2 class of sleep apnea. In this study, we perform classification of 2, 3, and 4 class of sleep apnea. In this research we also perform feature extraction with four techniques, i.e. time domain, geometrical with histogram, poincare and frequency domain. We evaluate which feature achieve the best performance for sleep apnea identification. In the end of study we expect to achieve the best accuracy of sleep apnea identification with machine learning method. We also perform two methods of classification, i.e. subject-specific scheme and subject-independent scheme. This is paper is organized as follow. Research methodology is explained in Section 2. The simulation results and discussion are explained in section 3, while the result of the paper is concluded in Section 4.

\section{RESEARCH METHOD}

This research focuses on HRV Features of ECG signaling to identify sleep apnea. We create classification model based on the data obtained from ECG signal. In this research, we identify the sleep apnea until 4 classes, i.e. non-sleep apnea, hypoapnea, obstructive apnea, and central apnea. Figure 1 shows the methodology flowchart of the research. The next subsection presents about the detail of every step in the flow chart, started from data collection, data pre-processing, feature extraction, classification model development and performance evaluation.

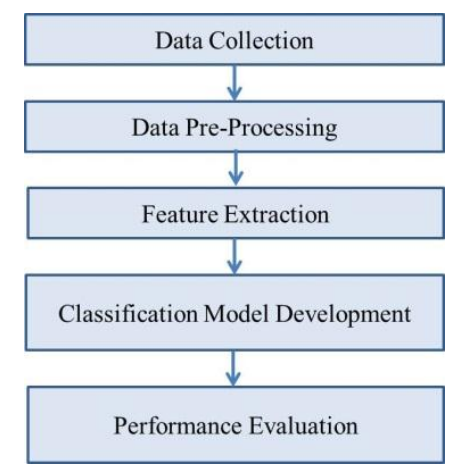

Figure 1. Research methodology

\subsection{Data collection}

At this stage the data collected where the data used is MIT-BIH Polysomnographic Database (slpdb) [12] obtained from https://physionet.org. This data contains a collection of recordings of several physiological signals of objects during sleep, ie ECG, EEG, blood pressure and respiratory signals, and calibration constants, the length of the recording, age, gender, and weight. The data used comes from male 
sleep records, aged 32-56 years old (average 43 years old), weight $89-152 \mathrm{~kg}$ (average $119 \mathrm{~kg}$ ). The data consists of 18 recordings of 16 people who have different durations of measurement. Then, every 30 seconds of duration, it was made into 1 sample data that will proceed to the pre-processing phase of the data. The data is about the RR interval and Sleep apnea annotations. Table 1 show samples of data which had been divided into 30 seconds and as data used to obtain HRV features. Figure 2 shows one of the signals from the MITBIH Polysomnographic Database (slp01a) data to be separated every 30 seconds.

Table 1. Sleep Apnea Datasets

\begin{tabular}{lcc}
\hline \multicolumn{1}{c}{ Data } & $\begin{array}{c}\text { Duration } \\
\text { (hour-minutes) }\end{array}$ & $\begin{array}{c}\text { Number of } \\
\text { samples }\end{array}$ \\
\hline slp01a & $2 \mathrm{H}$ & 240 \\
slp01b & $3 \mathrm{H}$ & 360 \\
slp02a & $3 \mathrm{H}$ & 360 \\
slp02b & $2 \mathrm{H} \mathrm{15} \mathrm{M}$ & 270 \\
slp03 & $6 \mathrm{H}$ & 720 \\
slp04 & $6 \mathrm{H}$ & 720 \\
slp14 & $6 \mathrm{H}$ & 720 \\
slp16 & $6 \mathrm{H}$ & 720 \\
slp32 & $5 \mathrm{H} \mathrm{20} \mathrm{M}$ & 640 \\
slp37 & $5 \mathrm{H} \mathrm{50} \mathrm{M}$ & 700 \\
slp41 & $6 \mathrm{H} \mathrm{30} \mathrm{M}$ & 780 \\
slp45 & $6 \mathrm{H} \mathrm{20} \mathrm{M}$ & 760 \\
slp48 & $6 \mathrm{H} \mathrm{20} \mathrm{M}$ & 760 \\
slp59 & $4 \mathrm{H}$ & 480 \\
slp60 & $5 \mathrm{H} \mathrm{55} \mathrm{M}$ & 710 \\
slp61 & $6 \mathrm{H} \mathrm{10} \mathrm{M}$ & 740 \\
slp66 & $3 \mathrm{H} \mathrm{40} \mathrm{M}$ & 440 \\
slp67x & $1 \mathrm{H} 17 \mathrm{M}$ & 154 \\
& Total of Sample & 10274 \\
\hline
\end{tabular}

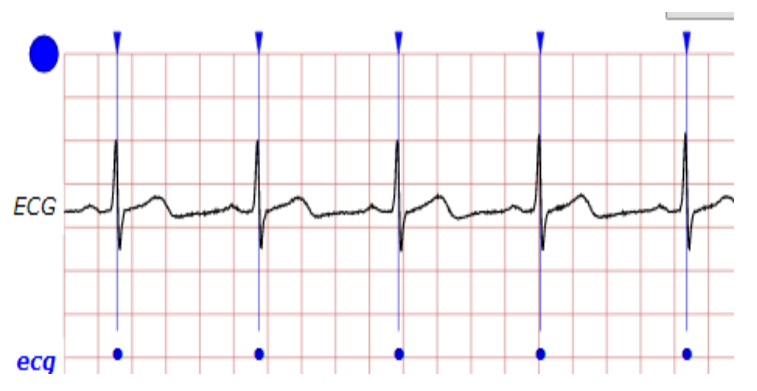

Figure 2. Sample of ECG signal used for sleep apnea identification

\subsection{Data pre-processing}

The pre-processing stage is the disposal stage of ECG recording data (RR intervals and sleep apnea annotations) that are out of sync in order to obtain the valid data. In this stage, we remove data slp03 and slp60 because the data are out of sync. Then all data from the pre-processing results is entered into the formulas to get the HRV features.

\subsection{Feature extraction}

In this process, data from pre-processing result is processed to obtain HRV features. We employ four techniques in this paper, i.e. time domain, geometrical, poincare, and frequency domain [13], [14]. The features contain SDNN, RMSDD, NN50, pNN50, Mean RR, HRV Index, SD1, SD2, SD1 / SD2, TP, VLF, LF, HF, LF / HF, LFnorm, HFnorm. Time Domain is derived from ECG Signal Data in the form of RR interval, frequency Domain of total spectrum of each beat separated in index to generate PSD. There are 18 features used in this research as shown by Table 2. We perform feature extraction using matlab. Figure 3 shows the value of feature extraction with time domain method. Figure 4 shows the results of feature extraction with frequency domain techniques, while Figure 5 shows the feature extraction results with poincare techniques. 
Table 2. HRV Features List

\begin{tabular}{|c|c|c|c|c|}
\hline No & Feature & Method & Description & Equation \\
\hline 1 & AVNN & Time Domain & The average of all NN (or RR) intervals, & $A V N N=\frac{1}{N} \sum_{j=1} R R_{j}$ \\
\hline 2 & SDNN & Time Domain & $\begin{array}{l}\text { The standard deviation of all NN (or RR) } \\
\text { intervals }\end{array}$ & $S D N N=\sqrt{\frac{1}{N-1} \sum_{j=1}^{N}\left(R R_{j}-\overline{R R}\right)^{2}}$ \\
\hline 3 & RMSSD & Time Domain & $\begin{array}{l}\text { The square root of the average of the sum of } \\
\text { the squares of differences between adjacent } \\
\text { RR intervals }\end{array}$ & $R M S S D=\sqrt{\frac{1}{N-1} \sum_{j=1}^{N-1}\left(R R_{j+1}-R R_{j}\right)^{2}}$ \\
\hline 4 & SDSD & Time Domain & $\begin{array}{c}\text { Standard deviation of differences between } \\
\text { adjacent RR intervals }\end{array}$ & - \\
\hline 5 & NN50 & Time Domain & $\begin{array}{l}\text { The count of adjacent NN (or RR) intervals } \\
\text { differences that are more than } 50 \mathrm{~ms}\end{array}$ & Number of $\left(R R_{j+1}-R R_{j}\right)>50$ \\
\hline 6 & pNN50 & Time Domain & $\begin{array}{l}\text { The division of NN50 by total of all RR } \\
\text { intervals minus one times } 100\end{array}$ & $p N N 50=\frac{N N 50}{N-1} \times 100$ \\
\hline 7 & $\begin{array}{l}\text { HRV } \\
\text { Triangular } \\
\text { Index }\end{array}$ & Geometrical & $\begin{array}{l}\text { The total number of RR intervals divided by } \\
\text { the peak of histogram created from RR } \\
\text { intervals data with } 7.8125 \text { bin size }\end{array}$ & - \\
\hline 8 & SD1 & Poincare & $\begin{array}{l}\text { The standard deviation of points } \\
\text { perpendicular to the axis of line-of-identity }\end{array}$ & $S D 1^{2}=\frac{1}{2} S D S D^{2}$ \\
\hline 9 & SD2 & Poincare & $\begin{array}{l}\text { The standard deviation of points along the } \\
\text { axis of line-of-identity }\end{array}$ & $S D 2^{2}=2 S D N N^{2}-\frac{1}{2} S D S D^{2}$ \\
\hline 10 & $\begin{array}{l}\text { SD1SD2 } \\
\text { Ratio }\end{array}$ & Poincare & Ratio of SD1 and SD2, & SD1 SD2 Ratio $=\frac{S D 1}{S D 2}$ \\
\hline 11 & S & Poincare & Area of ellipse & $S=\pi \times \mathrm{SD} 1 \times \mathrm{SD} 2$ \\
\hline 12 & $\mathrm{TP}$ & $\begin{array}{l}\text { Frequency } \\
\text { Domain }\end{array}$ & Total power & - \\
\hline 13 & VLF & $\begin{array}{l}\text { Frequency } \\
\text { Domain }\end{array}$ & Total power of 0 to $0.04 \mathrm{~Hz}$ & - \\
\hline 14 & $\mathrm{LF}$ & $\begin{array}{l}\text { Frequency } \\
\text { Domain }\end{array}$ & Total power of 0.04 to $0.15 \mathrm{~Hz}$ & - \\
\hline 15 & $\mathrm{HF}$ & $\begin{array}{l}\text { Frequency } \\
\text { Domain }\end{array}$ & Total power of 0.15 to $0.4 \mathrm{~Hz}$ & - \\
\hline 16 & $\begin{array}{l}\text { LFHF } \\
\text { Ratio }\end{array}$ & $\begin{array}{l}\text { Frequency } \\
\text { Domain }\end{array}$ & Ratio of LF and HF & - \\
\hline 17 & LFnorm & $\begin{array}{l}\text { Frequency } \\
\text { Domain }\end{array}$ & Normalized LF & $L F$ norm $=\frac{L F}{T P-V L F}$ \\
\hline 18 & HFnorm & $\begin{array}{l}\text { Frequency } \\
\text { Domain }\end{array}$ & Normalized HF & HFnorm $=\frac{H F}{T P-V L F}$ \\
\hline
\end{tabular}

Time Domain and Geometrical Result

\begin{tabular}{lrr}
\hline Variable & Units & Value \\
\hline Mean RR & $(\mathrm{ms})$ & 923.6 \\
STD RR (SDNN) & $(\mathrm{ms})$ & 44.1 \\
RMSSD & $(\mathrm{ms})$ & 22.5 \\
NN50 & $($ count) & 1 \\
pNN50 & $(\%)$ & 3.2 \\
RR trianqular index & & 8.000 \\
\hline
\end{tabular}

Figure 3. Result of time domain \& geometrical features

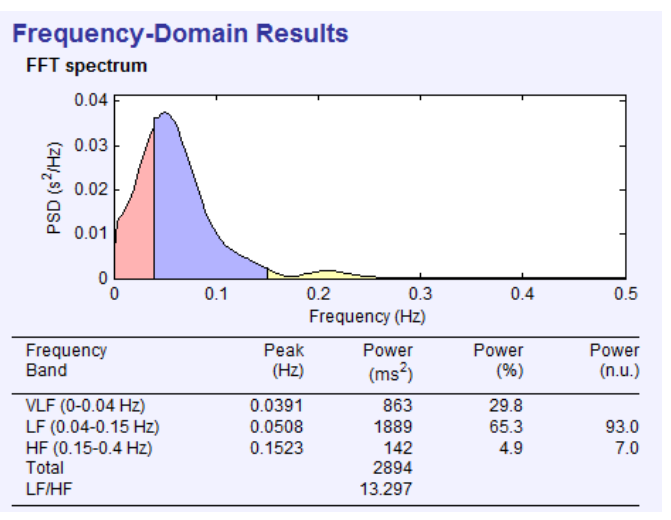

Figure 4. Result of frequency domain techniques 


\begin{tabular}{lcc}
\hline Variable & Units & Value \\
\hline Poincare plot & & \\
SD1 & $(\mathrm{ms})$ & 16.2 \\
SD2 & $(\mathrm{ms})$ & 60.2 \\
SD1/SD2 & $(\mathrm{ms})$ & 0.269
\end{tabular}

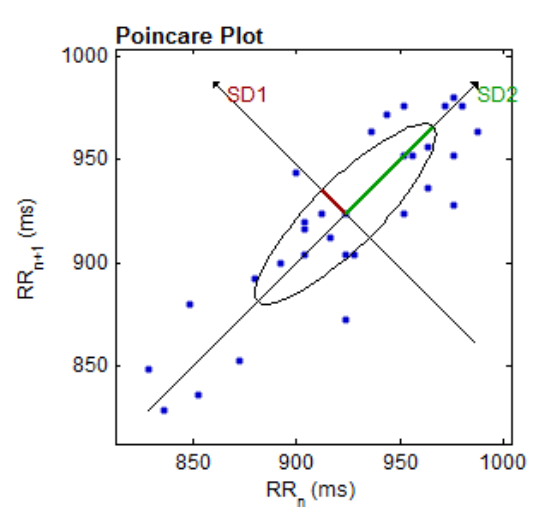

Figure 5. Feature Extraction with poincare techniques

\subsection{Classification process}

In this paper, we evaluate following four classification methods in classifying sleep apnea. All of these method are the most well-known classification method [15], i.e.:

a. Artificial Neural Network (ANN) is an algorithm where there is a network of small process units that are modeled or constructed based on the behavior of human neural networks [16]. The function of the neural network is the classification of patterns, pattern mapping obtained from inputs into new patterns of output, mapping of similar patterns, and problem solvers [17]. This ANN is commonly applied for noise identification, wave analysis, speed analysis, reservoir characterization and etc.

b. The Naive Bayes Classifier is a simple probability classification which uses Bayes's theorem with high independency assumptions [18]. Since variables are assumed to be independent so that the variance of a variable in a class is required to determine the classification. The advantage of using this method is that it requires only a few amount of training data to determine the required estimation parameters in the classification process.

c. K-Nearest Neighbor (K-NN) is one of a supervised algorithm where grouping of an object is classified by the majority of the nearest category. The goal is to classify new objects based on the attributes and samples of the data[19]. The K-NN algorithm works based on the minimum distance from the new data to the samples data to determine the K number of nearest neighbors. From here we obtain the majority value used as the predicted result of the new data.

d. Support Vector Machine (SVM) is a technique for making predictions in both classification and regression cases. The intention of SVM is to find an optimal separating hyperplane (OSH), which is the largest margin between the two datasets. It can be found by maximizing the margin between the classes. Firstly, SVM transforms input data into a higher dimensional space by using a kernel function. Then, SVM constructs a linear OSH between the two classes in the transformed space. The nearest data vectors to the constructed line in the transformed space are called as the support vectors [10].

\subsection{Performance evaluation}

The data is divided into 2: $70 \%$ training data and $30 \%$ data testing to build the model of the classification to be tested. The result data of the extract feature will be randomized to classify the ANN, KNN, N-Bayes and linear SVM classification methods. We perform the classification with rapidminer. The experiments are conducted several times for each of the following classes:

a. 2 classes: Sleep apnea and non-sleep apnea

b. 3 classes: non-sleep apnea/hypo-apnea/obstructive-apnea

c. 4 classes: non-sleep apnea/hypo-apnea/obstructive-apnea/central-apnea

We perform classification using subject-specific scheme and subject-independent scheme [20]. The difference between them is about the selection of training and testing sets. In subject-specific scheme, the training and testing sets are selected from the same record before being inputted to classifier model. On the other hand, in subject-independent scheme, the training and testing sets are combined from all records. The 
subject independent scheme is more practical [20]. However subject specific scheme more useful for multinight investigations and evaluating the importance of each feature for sleep staging.

\section{RESULTS AND DISCUSSION}

Firstly, we perform classification of sleep apnea for 2 classes of classification. As mentioned in Section 2, we evaluate the performance of 4 classification method, i.e. ANN, KNN, N-Bayes, linear SVM. Table 3 shows the result of 2 classes of classification with subject-specific scheme. Firstly, we calculate the accuracy for every subject (total 16 subjects used in this experiment) and perform mean operation at the end to compute the average accuracy. Table 3 shows that the SVM linear shows the most superior performance among them. SVM linear achieves $75.8 \%$ accuracy, almost $3 \%$ more than the second one, i.e. ANN.

Table 3. Accuracy for 2 Classes Classification with Subject-specific Scheme

\begin{tabular}{ccccc}
\hline \multirow{2}{*}{ Data } & ANN & KNN & N-Bayes & SVM(linear) \\
\hline Slp01a & $77.78 \%$ & $77,78 \%$ & $76,39 \%$ & $83,33 \%$ \\
Slp01b & $86,11 \%$ & $79,63 \%$ & $87,96 \%$ & $86,11 \%$ \\
Slp02a & $82,41 \%$ & $69,44 \%$ & $64,81 \%$ & $82,41 \%$ \\
Slp02b & $85,00 \%$ & $80,00 \%$ & $72,50 \%$ & $91,25 \%$ \\
Slp03 & $80,00 \%$ & $64,29 \%$ & $81,90 \%$ & $76,67 \%$ \\
Slp04 & $81,94 \%$ & $71,30 \%$ & $84,72 \%$ & $85,65 \%$ \\
Slp14 & $71,03 \%$ & $61,21 \%$ & $67,29 \%$ & $72,90 \%$ \\
Slp16 & $62,02 \%$ & $53,37 \%$ & $66,35 \%$ & $64,90 \%$ \\
Slp32 & $82,81 \%$ & $73,96 \%$ & $72,40 \%$ & $83,33 \%$ \\
Slp37 & $84,69 \%$ & $79,90 \%$ & $77,99 \%$ & $85,17 \%$ \\
Slp48 & $73,25 \%$ & $62,72 \%$ & $71,05 \%$ & $73,68 \%$ \\
Slp59 & $56,72 \%$ & $49,64 \%$ & $47,45 \%$ & $48,18 \%$ \\
Slp60 & $65,24 \%$ & $56,67 \%$ & $60,48 \%$ & $63,33 \%$ \\
Slp61 & $60,19 \%$ & $60,65 \%$ & $73,15 \%$ & $68,52 \%$ \\
Slp66 & $68,94 \%$ & $64,39 \%$ & $75,76 \%$ & $81,06 \%$ \\
Slp67x & $65,22 \%$ & $47,83 \%$ & $73,91 \%$ & $67,39 \%$ \\
Mean & $73,96 \%$ & $65,80 \%$ & $72,13 \%$ & $75,87 \%$ \\
\hline
\end{tabular}

Figure 6 and Figure 7 shows the result of classification for 2, 3, and 4 classes with subject-specific scheme and subject-independent scheme, respectively. We can observe in Figure 6 that SVM achieves the best performance among all method with $75.87 \%, 73.58 \%$, and $71.58 \%$ for 2,3 , and 4 classses, respectively. While ANN becomes the second best with performance lower about 1-2\% compared to the SVM. Interestingly, as shown by Figure 7, it shows that the performance of ANN slightly better than SVM for 3 and 4 classes classification. Based on this result it shows that in overall, for subject-independent scheme and subject-specific scheme, SVM shows the best performance among them. The reason maybe due to the nature of SVM that performs data selection before doing classification. There it results in better accuracy performance. However, the anomaly in subject independent scheme with 3 and 4 classes classification shows the accuracy result sometimes also influenced by the distribution of data training and testing.

From Figure 6 and Figure 7, we can also evaluate how good the generalization capability of each method is. For example, these results shows that ANN algorithm has good generalization capability because the decrease of accuracy between subject-specific and subject independent scheme is the lowest and most constant among all method, i.e. around 4\% for 2, 3, 4 classes of classification. SVM eventhough shows the best performance in overall as explained in the previous paragraph, it suffers $8 \%$ decrease of accuracy in 3 and 4 classes of classification. While N-Bayes shows the worst generalization capability by suffers almost $20 \%$ of decrease in 4 classes of classification accuracy.

On the other experiments, we evaluate which features extraction techniques has better performance for classification in every classification method. The result is shown by Figure 8 . The time domain features shows dominant performance with ANN and SVM method. While, poin care techniques and frequencydomain techniques shows the best performance with N-bayes method and KNN method, respectively. This results means every feature extraction method has different performance in every classification method. Howevery, for sleep apnea case, The time domain features shows the best performances in overall. 


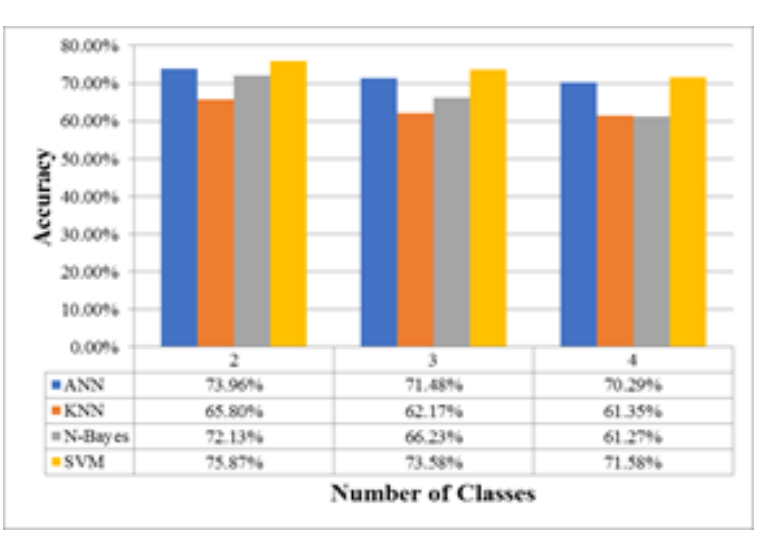

Figure 6. Accuracy performance evaluation with subject-specific scheme

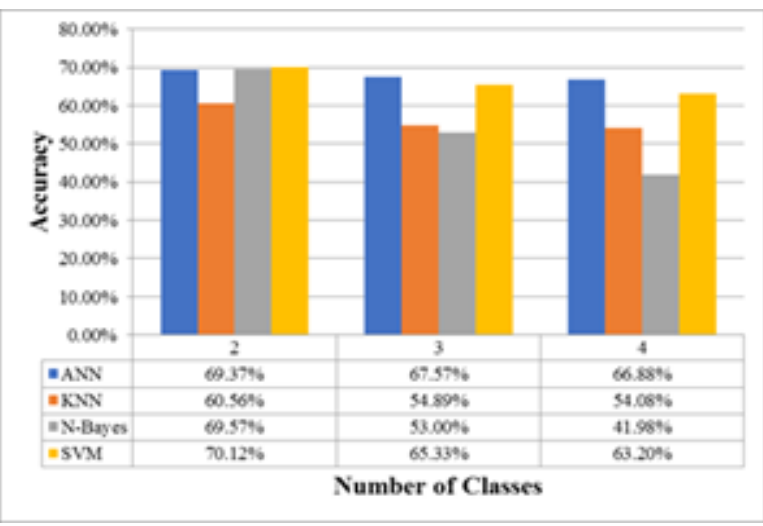

Figure 7. Accuracy performance evaluation with subject-independent scheme

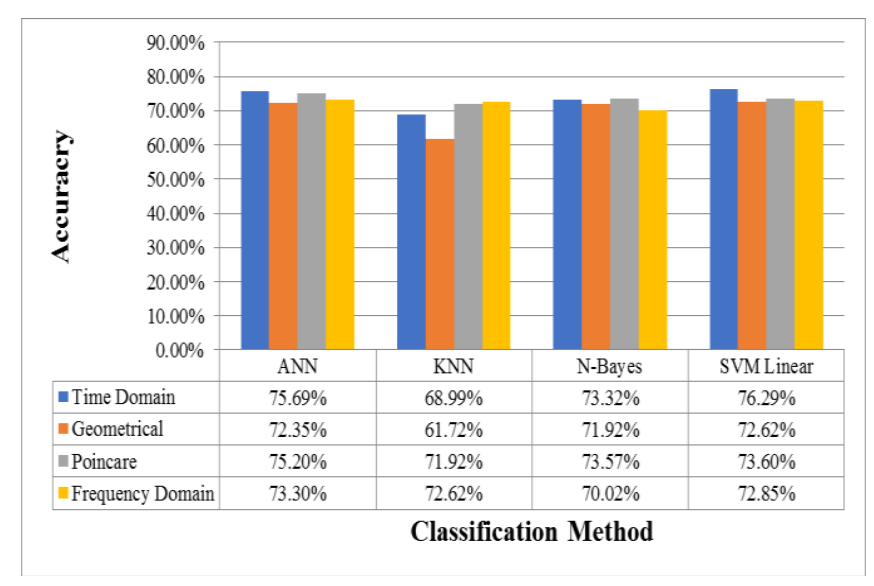

Figure 8. Feature extraction method comparison

Finally, we perform a comparison with the existing paper and can be seen through Table 4 . The comparison is performed for classification with 2 classes. Table 4 shows that our results outperforms the result of other work by Tilmaz and Erazo with an accuracy of $75.89 \%$. For the future, the sleep apnea classification model that uses the HRV feature of ECG signal for time domain, poincare, geometrical, and frequency domain can produce better accuracy. Then it can produce real-time sleep aids from the ECG signal results when a person sleeps.

Table 4. Comparison with other Works

\begin{tabular}{cccc}
\hline Author & Year & Approach & Accuracy Result \\
\hline Yilmaz [21] & 2010 & kNN, QDA, SVM & $74.4 \%$ \\
Erazo [22] & 2014 & ANN \& SVM & $55.94 \%$ \\
Proposed & 2017 & ANN \& SVM Linear & $74.85 \%$ \\
\hline
\end{tabular}

\section{CONCLUSION}

In this paper, we have performed evaluation of sleep apnea identification using HRV features of ECG signal. We have performed comparison of which classification method performs better and what HRV features shows the best performance for sleep apnea identification. We perform the classification for 2, 3, and 4 classes of sleep apnea. Our simulation results shows that the linear SVM achieves the best accuracy compared to the three other methods. While, the ANN method shows the best generalization capability among all method. On the other results, the time domain features shows the most dominant performance among the HRV features. At the end, we shows how our works shows slightly better performance compared to the other references. For the future research, we are going to employ classification method developed in 
this paper into sleep apnea monitoring prototype. We are going to build the portable and contactless prototype that we hope can help people to do self monitoring of sleep apnea symptoms at home.

\section{ACKNOWLEDGEMENTS}

This research was supported by Bina Nusantara University.

\section{REFERENCES}

[1] Wuen KC bin Mohd Saad WH, et al., "Study on the Effect of the Ambient Temperature toward the Quality of Sleep", Int J Electr Comput Eng., vol. 7, no. 6, pp. 2986-92, 2017.

[2] Surantha N., et al., "Internet of things for Sleep Quality Monitoring System: A survey", Knowledge, Information and Creativity Support Systems (KICSS), 2016 11th International Conference on., pp. 1-6, 2016.

[3] Morton A. J., "Circadian and Sleep Disorder in Huntington's Disease", Exp Neurol., vol. 243, pp. 34-44, 2013.

[4] Qaseem A., et al., "Diagnosis of Obstructive Sleep Apnea in Adults: A Clinical Practice Guideline from the American College of Physicians", Ann Intern Med., vol. 161, no. 3, pp. 210-20, 2014.

[5] Cowie M. R., et al., "Adaptive Servo-ventilation for Central Sleep Apnea in Systolic Heart Failure", $N$ Engl J Med., vol. 373, no. 12, pp. 1095-105, 2015.

[6] McEvoy R. D., et al., "CPAP for Prevention of Cardiovascular Events in Obstructive Sleep Apnea”, N Engl J Med., vol. 375, no. 10, pp. 919-31, 2016.

[7] Chirinos J. A., et al., "CPAP, weight loss, or both for Obstructive Sleep Apnea”, N Engl J Med., vol. 370, no. 24, pp. 2265-75, 2014.

[8] Almazaydeh L., et al., "Obstructive Sleep Apnea Detection using SVM-based Classification of ECG Signal Features", Engineering in Medicine and Biology Society (EMBC), 2012 Annual International Conference of the IEEE, pp. 4938-41, 2012.

[9] Yilmaz B., et al., "Sleep Stage and Obstructive Apneaic Epoch Classification using Single-lead ECG”, Biomed Eng Online, vol. 9, no. 1, p. 39, 2010.

[10] Varon C., et al., "Sleep Apnea Classification using Least-squares Support Vector Machines on Single Lead ECG", pp. 5029-32, 2013.

[11] Isa S. M., et al., "Sleep Apnea Detection from ECG signal: analysis on optimal features, principal components, and nonlinearity," Bioinformatics and Biomedical Engineering, (iCBBE) 5th International Conference, pp. 1-4, 2011.

[12] Goldberger A. L., et al., "PhysioBank, PhysioToolkit, and PhysioNet: Components of a new Research Resource for Complex Physiologic Signals”, Circulation, vol. 101, no. 23, pp. E215-20, 2000.

[13] Tarvainen M. P., et al., "Kubios HRV--Heart Rate Variability Analysis Software", Comput Methods Programs Biomed., vol. 113, no. 1, pp. 210-20, 2014.

[14] Godoy E., et al., "Time-Domain, Frequency Domain and Non-Linear Measurements in Neonates 骞Heart Rate Variability with Clinical Sepsis Sepsis, a Critical Bacterial Infection of the Bloodstream, is a Serious Cause of Illness in Neonatal Period in both Premature and at ter", Computing in Cardiology Conference (CinC), pp. 429-32, 2014.

[15] Abdar M., et al., "Comparing Performance of Data Mining Algorithms in Prediction Heart Diseases", Int J Electr Comput Eng., vol. 5, no. 6, pp. 1569-76, 2015.

[16] Moraes R., et al., "Document-level Sentiment Classification: An Empirical Comparison between SVM and ANN", Expert Syst Appl., vol. 40, no. 2, pp. 621-33, 2013.

[17] Prasad S. V. S., et al., "Comparison of Accuracy Measures for RS Image Classification using SVM and ANN Classifiers", Int J Electr Comput Eng., vol. 7, no. 3, pp. 1180-7, 2017.

[18] Zhou X., et al., "Detection of Pathological Brain in MRI Scanning based on Wavelet-entropy and Naive Bayes Classifier", International Conference on Bioinformatics and Biomedical Engineering, pp. 201-9, 2015.

[19] Sutton O., "Introduction to k nearest Neighbour Classification and Condensed nearest Neighbour Data Reduction the k nearest Neighbours Algorithm", pp. 1-10, 2012.

[20] Xiao M., et al., "Sleep Stages Classification based on Heart Rate Variability and Random Forest", Biomed Signal Process Control, vol. 8, no. 6, pp. 624-33, 2013.

[21] Yilmaz B., et al., "Sleep stage and obstructive apneaic epoch classification using single-lead ECG", Biomed Eng Online, vol. 9, no. 1, p. 39, 2010.

[22] Ríos S. A. and Erazo L., "An Automatic Apnea Screening Algorithm for Children”, Expert Syst Appl., vol. 48, pp. 42-54, 2016.

\section{BIOGRAPHIES OF AUTHORS}

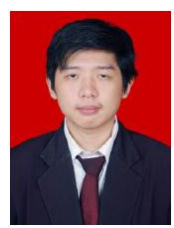

Billy Sulistyo received the bachelor degree in Computer Engineering in 2013. From 2015 until now, he is graduate student of Information Technology in Bina Nusantara University. His research interest is network design, network performance analysis, and machine learning. 

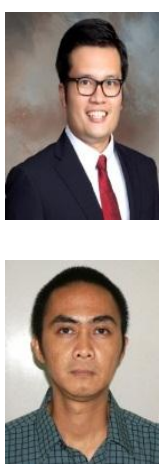

Nico Surantha received his B.Eng (2007) and M.Eng. (2009) from Institut Teknologi Bandung, Indonesia. He received his PhD degree from Kyushu Institute of Technology, Japan, in 2013. Currently, he serves as an assistant professor in Computer Science Department, Binus Graduate Program, Bina Nusantara University. His research interest includes wireless communication, health monitoring, network design, digital signal processing, system on chip design, and machine learning. He is an IEEE member.

Sani M. Isa is a lecturer and researcher in Computer Science Department, BINUS Graduate Program Master of Computer Science. He has some experiences in teaching and research in remote sensing and biomedical engineering areas. He got his doctoral degree in Computer Science from University of Indonesia. $\mathrm{He}$ is also received his master degree in Computer Science from University of Indonesia as well as bachelor degree from Padjadjaran University, Bandung, Indonesia. 